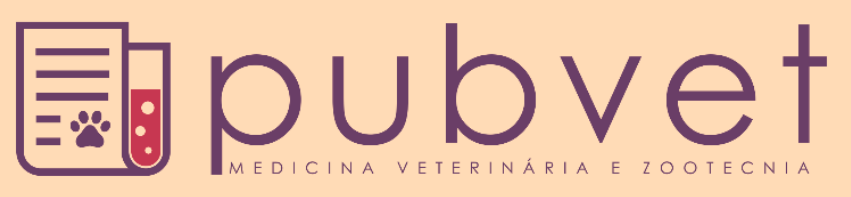

https://doi.org/10.31533/pubvet.v14n9a641.1-5

\title{
Urolitíase em cão da raça Pug
}

\author{
Marcela Lopes Ferraz ${ }^{1}$, Anne Cristine Eurides ${ }^{1}{ }^{\ominus}$, Beatriz Rodrigues Machado ${ }^{1}$, Luis Henrique \\ Silveira Baioco ${ }^{1}$, Anderson Diniz Rossi ${ }^{2}$, Vanessa Schnirman Wiecheteck ${ }^{2}$ \\ ${ }^{I}$ Aluno/a do Centro de Ensino Superior dos Campos Gerais - CESCAGE - Ponta Grossa-PR, Brasil. \\ ${ }_{2}^{2}$ Médico/a Veterinário do Conselho Regional de Medicina Veterinária do Paraná - CRMV-PR, Ponta Grossa -PR, Brasil. \\ Autor para correspondência, E-mail: marcelalopesferraz@hotmail.com
}

Resumo. A obstrução uretral, parcial ou total é uma das principais emergências em pequenos animais. Podendo ser causada por urólitos, neoplasia, granuloma ou decorrente de traumas penianos em machos. O perfil epidemiológico da doença mostra que esta ocorre com mais frequência em cães machos, sendo rara em filhotes e comum em animais entre 6 e 11 anos de idade. Os sinais clínicos mais comuns são hematúria, anúria, disúria e incontinência urinária. O presente relato tem como objetivo descrever a ocorrência desta patologia em um cão, macho, idade 2 anos, raça Pug, que foi submetido a uma desobstrução uretral e cistotomia para retirada de urólitos.

Palavras chave: cão, desobstrução, hematúria, urólitos

\section{Urolithiasis in a Pug dog}

Abstract. Urethral obstruction, partial or total, is one of the main emergencies in small animals. It can be caused by uroliths, neoplasia, granuloma or due to penile trauma in males. The epidemiological profile of the disease shows that it occurs more frequently in male dogs, being rare in puppies and common in animals between 6 and 11 years of age. The most common clinical signs are hematuria, anuria, dysuria and urinary incontinence. The present report aims to describe the occurrence of this pathology in a dog, male, age 2 years, Pug breed, who underwent urethral clearance and cystotomy to remove uroliths.

Keywords: dog, clearance, hematúria, uroliths

\section{Urolitiasis em um perro Pug}

Resumen. La obstrucción uretral, parcial o total, es una de las principales emergencias en animales pequeños. Puede ser causada por urolitos, neoplasia, granuloma o por traumatismo penianos en machos. El perfil epidemiológico de la enfermedad muestra que ocurre con mayor frecuencia en perros machos, siendo rara en cachorros y común en animales entre 6 y 11 años de edad. Los signos clínicos más comunes son hematuria, anuria, disuria e incontinencia urinaria. El presente informe tiene como objetivo describir la aparición de esta patología en un perro, macho, de 2 años de edad, raza Pug, que se sometió a una desobstrucción uretral y una cistotomía para eliminar los urolitos.

Palabras clave: perro desobstrucción, hematúria, urolitos

\section{Introdução}

A obstrução uretral, parcial ou total é uma das emergências mais comuns em medicina veterinária. A afecção pode ser causada por urólitos, neoplasia, granuloma e constrição após evento traumático ou fratura do pênis (Downs \& Bjorling, 1987). Na maioria das vezes, entretanto, está associada com urólitos 
que se alojam próximo ao óstio peniano, na transição entre a uretra peniana e pélvica ou no arco isquiático (Downs \& Bjorling, 1987; Vargas, 2008).

As principais raças acometidas são o Schnauzer miniatura, Lhasa apso, Yorkshire terrier, Bichon frise, Shitzu e Poodle (Osborne et al., 1995; Osborne et al., 1999). Os urólitos mais encontrados em cães são os de fosfato amoníaco magnesiano (estruvita) e oxalato de cálcio (Oyafuso, 2008).

O sistema urinário dos animais domésticos tem a habilidade de formar urina concentrada, sendo uma de suas funções a eliminação dos resíduos corporais na forma líquida (Senior \& Finlayson, 1986). Quando a urina se torna supersaturada com sais dissolvidos, estes podem precipitar-se para formar cristais. Se estes cristais não forem excretados, podem agregar-se em concreções sólidas, conhecidas então como cálculos.

A urolitíase é uma das principais causas de formações de cálculos no trato urinário dos animais domésticos e refere-se ao fato de haver cálculos ou urólitos nos rins, ureter, bexiga ou uretra (Fossum, 2014). Independente do processo de formação, os urólitos são caracterizados pelo potencial de alterar a fisiologia do trato urinário (․ J. Ettinger et al., 2017; Feldman, 1997). Há uma série de fatores que contribuem para a formação dos urólitos, como o $\mathrm{pH}$ da urina, consumo reduzido de água e tipo de dieta do animal. A supersaturação da urina com sais, combinada a um alto aporte de minerais e proteínas na dieta é um dos fatores primários para formação de cálculos (G. Grauer, 2015).

O objetivo deste trabalho foi relatar um caso clínico de urolitíase ocorrido em uma clínica veterinária em Ponta Grossa.

\section{Relato do caso}

Cão macho, denominado Paçoca, 2 anos, raça Pug (Figura 1). O proprietário chegou à clínica com queixa de que desde o início do dia o animal estava apresentando dificuldade e posição de esforço para urinar, excretando quantidades muito pequenas de urina. Após a anamnese completa e observação, em função da dieta suspeitou-se de obstrução uretral, sendo realizada então sondagem. Durante o procedimento saíram alguns urólitos que impediram a desobstrução completa, então em contato com o proprietário informou-se que seria necessário o procedimento cirúrgico como tratamento de escolha. Baseado nos sinais clínicos característicos da doença e anamnese do animal, foi possível estabelecer o diagnóstico de urolitíase.

Realizou-se então cistocentese para promover alívio imediato e temporário, observando hematúria no conteúdo retirado e a presença de 8 cálculos na uretra sendo um "plug" (Figura 2).

Após o procedimento o animal permaneceu com a sonda. Durante o internamento o animal foi tratado conforme a necessidade do caso e os fármacos de escolha foram: Omeprazol por meio da dose de $2 \mathrm{~mL}$, IV, 2 vezes ao dia (BID), a fim de proteger a mucosa gástrica, visto que o paciente receberia grande quantidade de medicamentos. O analgésico de eleição foi o Cloridrato de Tramadol com dose de $0,9 \mathrm{~mL}$, IM, BID e, Dipirona principalmente com finalidade antipirética, sendo utilizada a dose de $0,8 \mathrm{~mL}$, IM, BID. O anti-inflamatório empregado foi a Dexametasona sendo usada dose de 0,5mL, IM, 1 vez ao dia, com propósito de evitar processos inflamatórios no canal uretral decorrente da cistocentese. Como o animal estava com a sonda, esta foi lavada com $20 \mathrm{~mL}$ de soro fisiológico de $8 \mathrm{em} 8 \mathrm{~h}$.

Após a autorização do proprietário, o animal foi submetido à cistotomia, na qual se notou quantidade exorbitante de pequenos cálculos, que foram removidos da vesícula urinária (Figura 3). Seguido da cirurgia o paciente recebeu os medicamentos pós-operatórios e ficou em observação, recebendo alta após $24 \mathrm{~h}$ sem apresentar nenhuma complicação pós-cirúrgica. Foram prescritos os seguintes medicamentos para que o proprietário administrasse: Amoxicilina+Clavulanato de Potássio 250mg, com dose de $1 / 2$ comprimido, BID, durante 10 dias; Prednisolona 20mg dose $1 / 4$ do comprimido, BID, durante 4 dias. O retorno foi agendado para 90 dias após a alta, com o objetivo de realizar ultrassonografia e avaliar as estruturas do trato urinário.

\section{Discussão}

Os sinais clínicos de urolitíase variam conforme a localização, o tamanho e a quantidade de urólitos. Os animais acometidos podem permanecer assintomáticos. Sinais mais comuns são a polaciúria, disúria e hematúria, que se apresentaram no paciente em questão. Em casos de uretrólitos, pode ocorrer 
eliminação de urólitos pequenos e lisos à micção. A obstrução completa do fluxo urinário pode resultar em uremia pós-renal (Ettinger et al., 2017; Ettinger, 2003; Osborne et al., 1995; Osborne et al., 1999).

A alta incidência de urólitos e sua composição são influenciados diretamente por sexo, idade, raça, manejo nutricional, $\mathrm{pH}$ da urina e anomalias anatômicas. A dieta pode interferir de forma benéfica ou maléfica nas urolitíases de cães e gatos já que os alimentos influenciam diretamente no volume, $\mathrm{pH}$ e concentração da urina (Markwell et al., 1998).

Para o tratamento adequado e prevenção dessa afecção é necessário à identificação da causa, já que apresenta um alto nível de reincidências. O principal objetivo de uma dieta adequada no paciente com afecção urinária é a subsaturação da urina, através da diluição da mesma. A urina diluída tem menor concentração de minerais precursores dos cristais e aumento no volume urinário, o qual reduz as substâncias litogênicas, aumenta frequência de micção e favorece a remoção de qualquer cristal que possa ser formado (Monferdini \& Oliveira, 2009).

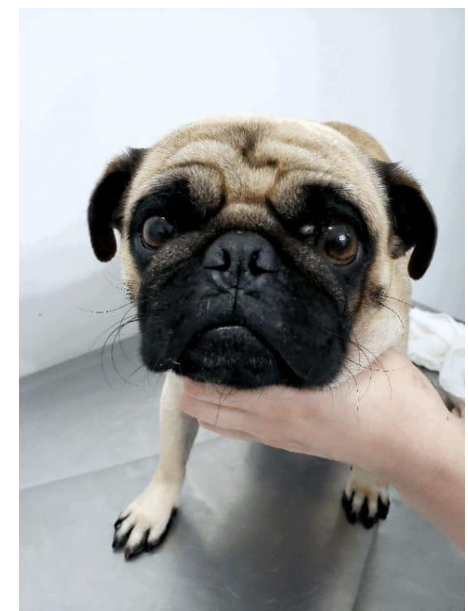

Figura 1. Cão macho, 2 anos, raça Pug apresentando dificuldade e posição de esforço para urinar

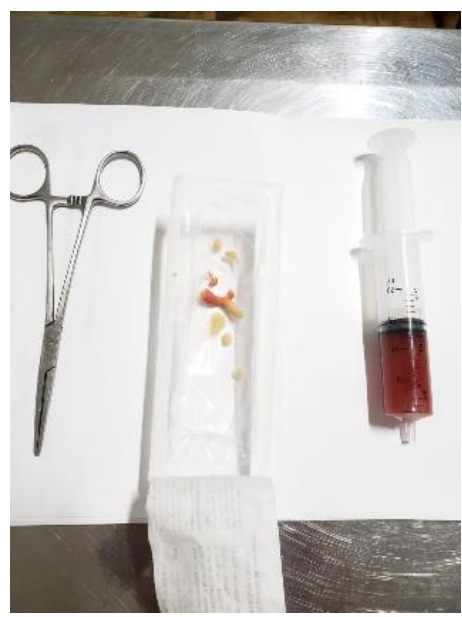

Figura 2. Conteúdo retirado após cistocentese.

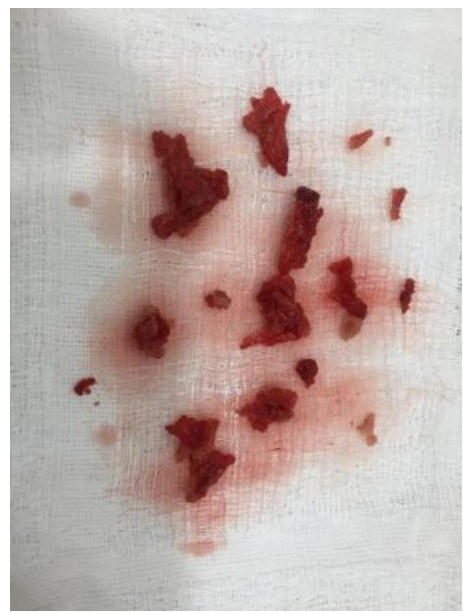

Figura 3. Fragmentos de urólitos retirados da vesícula urinária após a cistotomia.

No caso do paciente Paçoca, foi de extrema importância a mudança de dieta, visto que o proprietário ofertava ração com pouca quantidade de proteínas, gordura e fibras. A composição da ração interfere no surgimento da doença e também atua na prevenção de casos reincidivantes, pois está diretamente ligada ao $\mathrm{pH}$, volume e densidade específica da urina (Carciofi et al., 2009). É necessário o uso de ração adequada para afecções urinárias, cuja função é dissolver e evitar a formação de urólitos. A alimentação adequada é importante tanto a nível intestinal como na composição da urina. É importante ressaltar sobre a ingestão de água, quanto mais o animal ingerir menor a concentração de cristalóides na urina. Não é indicado a oferta de suplementos alimentares contendo cálcio, excesso de vitamina $\mathrm{C}$ e $\mathrm{D}$, pois vão aumentar a excreção de cálcio e/ou oxalato. Alguns autores relatam que uma dieta com baixo teor de sódio favorece a ingestão de água e, consequentemente, aumento do volume urinário, redução na densidade específica da água e aumento de miçcões (Monferdini \& Oliveira, 2009). Estudos demonstraram que um leve aumento de sódio na dieta não interfere na pressão sanguínea em cães e gatos (Elliott \& Lefebvre, 2009; Greco, 2006; Luckschander et al., 2004).

A obstrução uretral pode ser ocasionada por urólitos ou "plugs" uretrais (Ettinger et al., 2017). O tampão uretral ou "plug" geralmente é formado pela associação de uma matriz cristalina e uma matriz proteica que juntas formam os tampões mistos, apesar de existirem os de matriz única. A base da matriz cristalina pode ser constituída de oxalato de cálcio; porém, a mais comum, desde 1891, é a de estruvita (Bartges, 2012; Bartges \& Callens, 2015). Nos cães os cálculos uretrais são alojados com uma maior frequência no arco isquiático, ou em um local imediatamente caudal ao osso peniano. Em gatos a obstrução uretral geralmente é resultante da presença de muco localizado no terço distal da uretra (Slatter, 1998). Em casos de cálculos maiores, a bexiga urinária ou a uretra podem romper resultando em uma efusão abdominal ou acúmulo de líquido subcutâneo perineal e azotemia pós-renal. No cão, o local mais comum de presença de urólitos é na bexiga urinária, por isso geralmente nesses casos há sinais clínicos de cistite, como hematúria, 
polaciúria e disúria (Grauer, 2015), sinais clínicos compatíveis com o quadro do paciente deste relato. O diagnóstico de urolitíase envolve o histórico do paciente, exame físico, achados laboratoriais e exames de imagem (Grauer, 2015), no caso do paciente em questão não foram realizados exames complementares imediatos.

De acordo com Fossum (2014) o tratamento para urolitíase canina e felina varia conforme a composição do urólito e a sua localização, por métodos clínicos, terapêuticos e cirúrgicos. Basicamente, inclui avaliar e desfazer qualquer obstrução uretral e vesical quando necessário, e para isso, pode-se fazer a passagem de um cateter de pequeno calibre, deslocamento do cálculo por retrohidropropulsão ou cistocentese. O esvaziamento por uro-hidropropulsão pode remover pequenos cistólitos em cães machos e em fêmeas, sendo necessário o animal estar sob anestesia geral.

Condizente com a literatura, os tratamentos de escolha foram a cistocentese e cistotomia, obedecendo que o tratamento cirúrgico deve ser levado em consideração quando anormalidades anatômicas estão presentes, se a dissolução farmacológica não for possível, quando houver necessidade de cultura da mucosa do trato urinário ou quando os cálculos forem grandes a ponto de causar obstrução uretral Fossum (2014).

No caso relatado não foi possível fazer a dissolução dos cálculos através do uso de fármacos, pois o paciente já se encontrava em situação de emergência decorrente da obstrução e possibilidade de rompimento da vesícula urinária. O paciente em questão não demonstrou complicações pós-operatórias e apresentou resposta satisfatória ao tratamento.

\section{Conclusão}

A urolitíase é uma enfermidade comum em cães e gatos, considerando que algumas raças possuem predisposição é importante que cuidados básicos sejam tomados afim de evitar a doença, a observação do proprietário é de extrema importância para uma anamnese detalhada que contribui diretamente no diagnóstico, o animal em questão apresentou sinais repentinos, necessitando de atendimento emergencial. Diante disso é muito importante que o proprietário seja orientado sobre os devidos cuidados após o tratamento e a possibilidade de recidiva da doença, além da conduta clínica do profissional que deve prezar pela recuperação do paciente, perante dificuldades na realização do diagnóstico, exames complementares são essenciais, no caso relatado não foram realizados devido a sugesta dos sinais apresentados, somente foi agendado retorno para 90 dias afim de realizar ultrassonografia, sendo significativa a responsabilidade do proprietário para com seu animal de estimação, presenciando a consulta de retorno para que seja realizado acompanhamento e avaliação do paciente.

\section{Referências bibliográficas}

Bartges, J. W. (2012). Chronic kidney disease in dogs and cats. Veterinary Clinics: Small Animal Practice, 42(4), 669-692. DOI: https://doi.org/10.1016/j.cvsm.2012.04.008

Bartges, J. W., \& Callens, A. J. (2015). Urolithiasis. Veterinary Clinics: Small Animal Practice, 45(4), 747-768. DOI: https://doi.org/10.1016/j.cvsm.2015.03.001

Carciofi, A. C., Brunetto, M. A., Gomes, M. O. S., Teshima, E., \& Jeremias, J. T. (2009). Suporte nutricional parenteral no paciente crítico. Clínica Veterinária, 78, 52-60.

Downs, M. C., \& Bjorling, D. E. (1987). Traumatic diaphragmatic - hernias - a review of 1674 casos. Veterinary Surgery, 16, 87.

Elliott, D. A., \& Lefebvre, H. (2009). Textbook of veterinary internal medicine. In P. Pibot, V. Biourge, \& D. A. Elliott (Eds.), Encyclopedia of canine clinical nutrition royal canin (pp. 252-282). Royal Canin.

Ettinger, S. J., Feldman, E. C., \& Cote, E. (2017). Textbook of Veterinary Internal Medicine-eBook. Elsevier Health Sciences.

Ettinger, S. N. (2003). Principles of treatment for canine lymphoma. Clinical Techniques in Small Animal Practice, 18(2), 92-97. DOI: https://doi.org/10.1053/svms.2003.36622

Feldman, E. C. (1997). Tratado de medicina interna veterinária. In Moléstias do cão e do gato (Vol. 3).

Fossum, T. W. (2014). Cirurgia de pequenos animais (4th ed., Vol. 1). Elsevier Brasil.

Grauer, G. (2015). Manifestações clínicas dos distúrbios urinários; Urolítiase canina. In A. W. Nelson 
\& C. G. Couto (Eds.), Medicina Interna de Pequenos Animais (Issue 1). Elsevier Editora.

Grauer, G. F. (2015). Feline Struvite \& Calcium Oxalate Urolithiasis. Todays Vet Pract, 5(5), 14-20.

Greco, D. S. (2006). Pediatric endocrinology. Veterinary Clinics of North America: Small Animal Practice, 36, 549-556. DOI: https://doi.org/10.1016/j.cvsm.2005.12.005

Luckschander, N., Iben, C., Hosgood, G., Gabler, C., \& Biourge, V. (2004). Dietary NaCl does not affect blood pressure in healthy cats. Journal of Veterinary Internal Medicine, 18(4), 463-467. DOI: https://doi.org/10.1111/j.1939-1676.2004.tb02567.x

Markwell, P. J., Buffington, C. T., \& Smith, B. H. E. (1998). The effect of diet on lower urinary tract diseases in cats. The Journal of Nutrition, 128(12), 2753S-2757S. DOI: https://doi.org/10.1093/jn/128.12.2753s

Monferdini, R. P., \& Oliveira, J. (2009). Manejo nutricional para cães e gatos com urolitíase-Revisão bibliográfica. Acta Veterinaria Brasilica, 3(1), 1-4.

Osborne, C A, Lulich, J. P., Bartges, J. W., Unger, L. K., Thumchai, R., Koehler, L. A., Bird, K. A., \& Felice, L. J. (1995). Canine and feline urolithiasis: relationship of etiopathogenesis to treatment and prevention. Canine and Feline Nephrology and Urology, 37(12), 798-888.

Osborne, Carl A, Lulich, J. P., Polzin, D. J., Sanderson, S. L., Koehler, L. A., Ulrich, L. K., Bird, K. A., Swanson, L. L., Pederson, L. A., \& Sudo, S. Z. (1999). Analysis of 77,000 canine uroliths: perspectives from the Minnesota Urolith Center. Veterinary Clinics of North America: Small Animal Practice, 29(1), 17-38. DOI: https://doi.org/10.1016/j.cvsm.2008.09.011

Osborne, Carl A, Sanderson, S. L., Lulich, J. P., Bartges, J. W., Ulrich, L. K., Koehler, L. A., Bird, K. A., \& Swanson, L. L. (1999). Canine cystine urolithiasis: cause, detection, treatment, and prevention. Veterinary Clinics of North America: Small Animal Practice, 29(1), 193-211. DOI: https://doi.org/10.1016/s0195-5616(99)50011-9

Oyafuso, M. K. (2008). Estudo retrospectivo e prospectivo da urolitíase em cães. Universidade de São Paulo. DOI: https://doi.org/10.11606/d.10.2008.tde-12122008-104152

Senior, D. F., \& Finlayson, B. (1986). Initiation and growth of uroliths. The Veterinary Clinics of North America. Small Animal Practice, 16(1), 19-26. DOI: https://doi.org/10.1016/s0195-5616(86)50002-4

Slatter, D. H. (1998). Manual de cirurgia de pequenos animais (Vol. 2). Manole São Paulo.

Vargas, A. M. (2008). Emergências endócrinas. In M. M. Santos \& F. S. Fragata (Eds.), Emergência e terapia intensiva veterinária em pequenos animais - Base para o atendimento hospitalar (pp. 348356). Editora Roca.

Recebido: 15 de junho, 2020 .

Aprovado: 15 de julho, 2020.

Disponível online: 17 de agosto, 2020.

Licenciamento: Este artigo é publicado na modalidade Acesso Aberto sob a licença Creative Commons Atribuição 4.0 (CCBY 4.0), a qual permite uso irrestrito, distribuição, reprodução em qualquer meio, desde que o autor e a fonte sejam devidamente creditados. 